\title{
Brolucizumab and fluid in neovascular age-related macular degeneration (n-AMD)
}

\author{
Ashish Sharma ${ }^{1}$ Nilesh Kumar ${ }^{1}{ }^{1} \cdot$ Nikulaa Parachuri $^{1} \cdot$ Rohini Sharma $^{2} \cdot$ Francesco Bandello $^{3}$. \\ Baruch D. Kuppermann ${ }^{4} \cdot$ Carl D. Regillo ${ }^{5}$
}

Received: 3 February 2020 / Revised: 20 February 2020 / Accepted: 20 February 2020 / Published online: 4 March 2020

(c) The Royal College of Ophthalmologists 2020

Brolucizumab (Beovu, Novartis) was recently approved by the US FDA in October 2019 for the treatment of neovascular age-related macular degeneration (n-AMD). Brolucizumab is a $28 \mathrm{kDa}$ humanized single chain antibody fragment $(\mathrm{scFv})$ molecule that binds to all isoforms of VEGF-A and renders them ineffective [1].

Drying of fluid in the macula has been one of the major clinical end points for the management of n-AMD [2]. The clinical significance of fluid depends on its location \{intraretinal (IRF) or subretinal (SRF) or beneath the retinal pigment epithelium (sub-RPE)\} [3]. However, pathology that leads to the formation of fluid has a major role in determining the long term success of the treatment.

Brolucizumab was approved on the basis of the pivotal phase three clinical trial results of the HAWK and HARRIER studies. The visual gains were found to be noninferior to aflibercept at 1 year. It was further maintained at the end of 2 years. Visual gains of +6.6 letters in $6 \mathrm{mg}$ brolucizumab group compared with +6.8 letters with $2 \mathrm{mg}$ aflibercept in HAWK trial was maintained at 2 years with 5.9 letters and 5.3 letters, respectively. Similarly, a gain of +6.9 letters with $6 \mathrm{mg}$ brolucizumab, when compared with +7.6 letters with aflibercept in HARRIER, was maintained as 6.1 letters versus 6.6 letters, respectively.

Ashish Sharma

drashish79@hotmail.com

1 Lotus Eye Hospital and Institute, Avinashi Road, Coimbatore, TN, India

2 Department of Healthcare, The TIPS Global Institute, Coimbatore, Tamil Nadu, India

3 University Vita-Salute, Scientific Institute San Raffaele, Milano, Italy

4 Gavin Herbert Eye Institute, University of California, Irvine, CA, USA

5 The Retina Service of Wills Eye Hospital, Mid Atlantic Retina, Philadelphia, PA, USA
Although visual gain was the primary outcome through a 96-week follow-up period, fluid assessment in the form of central subfield thickness (CST) and the IRF/SRF was considered as a key, secondary outcome of these trials. At week $48,31.2 \%$ of patients in $6 \mathrm{mg}$ brolucizumab group had IRF/SRF compared with $44.6 \%$ in aflibercept in the HAWK trial and HARRIER showed IRF/SRF in $25.8 \%$ (brolucizumab) and $43.9 \%$ (aflibercept) patients. At 96 weeks, $24 \%$ and $37 \%$ of the patients had persistent IRF/ SRF in HAWK trials for $6 \mathrm{mg}$ brolucizumab and aflibercept, respectively. HARRIER showed a persistence rate of 24\% (brolucizumab) and 39\% (aflibercept) patients. At 96 weeks, $11 \%$ of patients in brolucizumab $6 \mathrm{mg}$ group had sub-RPE fluid compared with $15 \%$ in aflibercept group in HAWK. It was $17 \%$ and $22 \%$, respectively, in HARRIER trial. Furthermore, the mean change in CST from baseline graph by optical coherence tomography over time was found to be less variable as compared to the seesaw pattern observed with the aflibercept control arm. Lesser fluctuation in the CST is an indicator of better fluid control which could be beneficial for the health of the retinal tissue in the long term [4].

Superior fluid control across all the fluid types (IRF/SRF/ sub-RPE) needs further understanding about the probable mechanism. Earlier trials such as CATT and EXCITE have demonstrated that the presence of IRF is an indicator of a later decline in visual acuity [3, 5]. However, fluid should not be seen as an isolated entity, rather it should be analyzed in terms of the pathology causing it such as type 1, 2, or 3 macular neovascularization (MNV) [6]. The better control of IRF/SRF/sub-RPE fluid with brolucizumab might have contributed to the $>50 \%$ proportion of patients maintaining $\mathrm{q} 12 \mathrm{w}$ dosing through 1 year of follow-up [4]. The US FDA has approved a 12-week dosing schedule for the molecule after three monthly loading doses.

To further understand the superior fluid control with brolucizumab, it is important to understand the molecular structure. Brolucizumab was developed by grafting 
complementarity-determining regions of the novel antiVEGF antibody to a human scFv scaffold. ScFvs are the smallest functional unit of an antibody, allowing the delivery of a greater molar dose into the vitreous cavity compared with larger molecules [1]. This might provide better diffusion into tissue. The pre-clinical data revealed that the retina had a 2.2 times higher exposure to the molecule when compared with ranibizumab. RPE/Choroid complex also had 1.7 times higher exposure. Smaller size and higher molecular concentration might help to increase the duration of fluid control [4].

Fluid in n-AMD should be analyzed in relation to the original pathology. Sub-RPE fluid is predominantly caused by type $1 \mathrm{MNV}$ or polypoidal disease whereas SRF and IRF are more common in type $2 \mathrm{MNV}$. Type $2 \mathrm{MNV}$ would cause SRF in the early stages. After the external limiting membrane barrier is broken, fluid seeps into the intraretinal space. Therefore, the location of fluid indirectly indicates the stage of the disease. Presence of IRF leading to later decline in visual acuity refers to the advanced stage of the disease. However, type $3 \mathrm{MNV}$ \{retinal angiomatous proliferation (RAP) $\}$ can vary in this aspect [6].

The impact of brolucizumab on IRF/SRF and sub-RPE fluid has been analyzed in the HAWK and HARRIER studies. However, IRF and SRF were not analyzed in isolation. None of the prospective trials in the past have differentiated the types of fluid. Analysis of IRF, SRF, and sub-RPE fluid as separate entities might be more meaningful to understand the effect of brolucizumab on the theoretical concern of geographic atrophy due to enhanced drying. There are practical difficulties to find sufficient number of cases with compartmentalized fluid in trials. Real world large data sets might be helpful to help sort out these unanswered questions.

Landmark trials for n-AMD support regular monthly or bimonthly injections of anti-VEGF therapy to maintain vision [7-9]. This treatment regimen is difficult for patients to adhere to, as reflected in several real world treatment studies. Visual gains and the number of injections were significantly lower in real world compared to the gains demonstrated in well designed anti-VEGF therapy clinical trials $[10,11]$. Jeff et al. demonstrated that eyes with early persistent retinal fluid (after three loading doses of ranibizumab) would require frequent injections to have better outcomes. After a decade of anti-VEGF use in the management of n-AMD, the goal of treatment has shifted to explore the treatment strategies to reduce the injection burden by reducing the frequency of injections. Brolucizumab performance in clinical trials suggests that it might help retinal specialists address two continuing issues of anti-VEGF therapy: persistent retinal fluid despite treatment, and the burden on patients of frequent anti-VEGF injections. Brolucizumab seems to be a promising new generation anti-VEGF molecule based on the trial results. However, real world data are yet to confirm the same.

\section{Compliance with ethical standards}

Conflict of interest AS: Consultant: for Novartis, Allergan, Bayer and Intas. FB: Consultant: Allergan, Bayer, Boehringer- Ingelheim, Fidia Sooft, Hofmann La Roche, Novartis, NTC Pharma, Sifi, Thrombogenics, Zeiss. BDK: Clinical research: Alcon, Alimera, Allegro, Allergan, Apellis, Clearside, Genentech, GSK, Ionis, jCyte, Novartis, Regeneron, ThromboGenics; Consultant: Alimera, Allegro, Allergan, Cell Care, Dose, Eyedaptic, Galimedix, Genentech, Glaukos, Interface Biologics, jCyte, Novartis, Ophthotech, Regeneron, Revana, Theravance Biopharma. CDR: Consultant: Allergan, Chengdu Kanghong, Genentech/Roche, Novartis, Kodiak, Notal, Merck, Shire-Takeda, Adverum, Graybug, and Eyepoint and receives research support from Allergan, Chengdu Kanghong, Genentech/Roche, Novartis, Kodiak, Iveric, and Adverum. NK, NP, and RS: None.

Publisher's note Springer Nature remains neutral with regard to jurisdictional claims in published maps and institutional affiliations.

\section{References}

1. Sharma A, Kumar N, Kuppermann BD, Bandello F. Brolucizimab-leading an era of structural revolution for long-term VEGF suppression. Eye (Lond). 2019. https://doi.org/10.1038/ s41433-019-0583-z. [Epub ahead of print].

2. Jaffe GJ, Kaiser PK, Thompson D, Gibson A, Saroj N, Vitti R, et al. Differential response to anti-VEGF regimens in age-related macular degeneration patients with early persistent retinal fluid. Ophthalmology. 2016;123:1856-64.

3. Sharma S, Toth CA, Daniel E, Grunwald JE, Maguire MG, Ying GS, et al. Macular morphology and visual acuity in the second year of the comparison of age-related macular degeneration treatments trials. Ophthalmology. 2016;123:865-75.

4. Dugel PU, Koh A, Ogura Y, Jaffe GJ, Schmidt-Erfurth U, Brown DM, et al. HAWK and HARRIER: phase 3, multicenter, randomized, double-masked trials of brolucizumab for neovascular age-related macular degeneration. Ophthalmology. 2020; 127:72-84.

5. Simader C, Ritter M, Bolz M, Deák GG, Mayr-Sponer U, Golbaz I, et al. Morphologic parameters relevant for visual outcome during anti-angiogenic therapy of neovascular age-related macular degeneration. Ophthalmology. 2014;121:1237-45.

6. Spaide RF, Jaffe GJ, Sarraf D, Freund KB, Sadda SR, Staurenghi $\mathrm{G}$, et al. Consensus nomenclature for reporting neovascular age-related macular degeneration data: consensus on neovascular age-related macular degeneration nomenclature study group. Ophthalmology. 2019; pii: S0161-6420(19)32243-2.

7. Rosenfeld PJ, Brown DM, Heier JS, Boyer DS, Kaiser PK, Chung $\mathrm{CY}$, et al. Ranibizumab for neovascular age-related macular degeneration. N Engl J Med. 2006;355:1419-31.

8. Brown DM, Michels M, Kaiser PK, Heier JS, Sy JP, Ianchulev T, ANCHOR Study Group. Ranibizumab versus verteporfin photodynamic therapy for neovascular age-related macular degeneration: two-year results of the ANCHOR study. Ophthalmology. 2009;116:57-65.

9. Heier JS, Brown DM, Chong V, Korobelnik JF, Kaiser PK, Nguyen QD, et al. Intravitreal aflibercept (VEGF trap-eye) in wet age-related macular degeneration. Ophthalmology. 2012;119:2537-48. 
10. Holekamp NM, Liu Y, Yeh WS, Chia Y, Kiss S, Almony A, et al. Clinical utilization of anti-VEGF agents and disease monitoring in neovascular age-related macular degeneration. Am J Ophthalmol. 2014;157:825-33.
11. Holz FG, Tadayoni R, Beatty S, Berger A, Cereda MG, Cortez R, et al. Multi-country real-life experience of anti-vascular endothelial growth factor therapy for wet age-related macular degeneration. Br J Ophthalmol. 2015;99:220-6. 